\title{
XVI. On the extension of Budan's criterion for the imaginary roots, and a new method of effecting the separation of the nearly equal roots of a numerical equation
}

\author{
James R. Christie Esq.
}

To cite this article: James R. Christie Esq. (1842) XVI. On the extension of Budan's criterion for the imaginary roots, and a new method of effecting the separation of the nearly equal roots of a numerical equation, Philosophical Magazine Series 3, 21:136, 96-101, DOI: $10.1080 / 14786444208621492$

To link to this article: http://dx.doi.org/10.1080/14786444208621492

$$
\text { 曲 Published online: } 01 \text { Jun } 2009 .
$$

Submit your article to this journal ए

\section{Џll Article views: 1}

Q View related articles ¿ 
us, a generation which passes over our grave is sufficient to cause these titles not to be remembered; the facts are quoted, the authors are forgotten.

The works of Richter, as we have seen, belong to these two distinct classes, and if it is true that a few words should suffice to sum up the entire life of a celebrated man, that of Richter is altogether summed up in these words (taken from the $\mathrm{W}^{\top}$ isdom of Solomon, xi. 22) which he placed as an epigraph at the head of all of his works which treat of chemical proportions:

"God made all things, in measure, and number, and weight."

XVI. On the Extension* of Budan's Criterion for the Imaginary Roots, and a new Method of efferting the Separation of the nearly equal Roots of a numerical Equation. By JaMes R. Christie, Esq. $\dagger$

BUDAN has shown that his criterion of the presence of imaginary roots only fails when, in the pair of roots $\alpha \pm \beta \sqrt{-1}, \alpha$ is a positive proper fraction and $\beta$ is less than 5 , on account of the effect of his reciprocal transformation being that of converting these roots to the new form

$$
\frac{\alpha \pm \beta \sqrt{-1}}{\alpha^{2}+\beta^{2}} \text { or } \alpha_{1} \pm \beta_{1} V \overline{-1}
$$

wherein $\alpha_{1}$ must, in the failing case, be less than unity.

In the reduced reciprocal equation these roots become

$$
\alpha_{1}-1 \pm \beta_{1} \sqrt{-1}
$$

and they may, as before, be shown to be imaginary unless $\beta_{1}$ be less than $\cdot 5$.

If we suppose $\alpha$ to be not greater than $\beta$, then $\frac{1}{2 \beta}$ will be the least value of the fraction $\beta_{1}$; but $\beta$ is less than $\cdot 5$, consequently this value of $\beta_{1}$ must exceed unity. It appears therefore that, in the case of $\alpha$ not greater than $\beta$, the condition upon which the failure of the criterion depends, ceases to exist in the roots as they appear in the first reduced reciprocal equation. The same will hold true if $\alpha$ does not exceed $\beta \vee 3$, since the least value this condition allows for $\beta_{1}$ is $\cdot 5$.

Let us now see in what manner $\alpha$ and $\beta$ enter into the second reciprocal equation.

* It is proper to mention that, in 1840, I pointed out the practical application of this method, in an example which was casually brought under my notice, to my friend and colleague Mr. Davies, who considered the then crude remark as of sufficient importance to be inserted, with the example, in his last edition of Hutton's "Course of Mathematics."J. R. C.

+ Communicated by the Author. 
Supposing that the variations (which correspond to those of the original equation whose indications of roots, real or imaginary, we are attempting to discover by aid of the criterion) disappear from the equation in $\frac{1}{x}-(p+1)$, the roots in the immediately preceding equation will be of the form

$$
\begin{aligned}
& \frac{\alpha}{\alpha^{2}+\beta^{2}}-p \pm \frac{\beta \sqrt{-1}}{\alpha^{2}+\beta^{2}}, \\
& \frac{\alpha-p\left(\alpha^{2}+\beta^{2}\right) \pm \beta \sqrt{-1}}{\alpha^{2}+\beta^{2}},
\end{aligned}
$$

or

and in the second reciprocal equation

$$
\frac{\left(\alpha^{2}+\beta^{2}\right) \cdot\left\{\alpha-p\left(\alpha^{2}+\beta^{2}\right) \mp \beta \sqrt{-1}\right\}}{\left\{\alpha-p\left(\alpha^{2}+\beta^{2}\right)\right\}^{2}+\beta^{2}}
$$

which finally reduces to

$$
\frac{\alpha-p\left(\alpha^{2}+\beta^{2}\right)}{(1-p \alpha)^{2}+p^{2} \beta^{2}} \pm \frac{\beta \sqrt{-1}}{(1-p \alpha)^{2}+p \beta^{2}} .
$$

Now $p$ evidently represents the integer next less than $\frac{\alpha}{\alpha^{2}+\beta^{2}}$, to which, if we assume $\alpha$ greater than $\cdot 5$, the superior limit is 2 ; consequently, in this case, $p=1$, and the above expression becomes

or

$$
\frac{\alpha-\left(\alpha^{2}+\beta^{2}\right) \pm \beta \sqrt{-1}}{(1-\alpha)^{2}+\beta^{2}},
$$

(1)

making

$$
\alpha_{11} \pm \beta_{11} \sqrt{-1} \text {, }
$$

$$
\alpha_{11}=\frac{\alpha-\left(\alpha^{2}+\beta^{2}\right)}{(1-\alpha)^{2}+\beta^{2}} \text { and } \beta_{11}=\frac{\beta}{(1-\alpha)^{2}+\beta^{2}} \text {. }
$$

It is easily seen that $\beta_{11}$ decreases with the value of $\alpha$, and the lower limit of its value will therefore, in this case, be

$$
\frac{\beta}{25+\beta^{2}} \text {, }
$$

which decreases with the decrease of $\beta$; solving therefore the equation

$$
\frac{\beta}{.25+\beta^{2}}=\cdot 5 \text {, }
$$

we obtain $\beta=1 \pm .866$; and since $\beta$ must be less than $\cdot 5$, we have $\beta=\cdot 134$ as the value which $\beta$ cannot exceed if $\beta_{11}$ is less than $\cdot 5$.

Should $\beta_{11}$ be less than $\cdot 5$ ( $\alpha_{1}$ and $\alpha_{11}$ being greater than $\cdot 5$ ) Phil. Mag. S. 3. Vol. 21. No. 136. Aug. 1842. 
two more reciprocal transformations will give $\beta_{I V} \sqrt{-1}$ as the imaginary part of the corresponding pair of roots, $\beta_{\mathrm{IV}}$ depending in value on $\beta_{11}$ as $\beta_{11}$ does upon $\beta$; we get therefore, from the equation $\beta_{11}=\cdot 134$, the value

$$
\beta=\cdot 033
$$

as that which exceeds all values of $\beta$ which can make $\beta_{\mathrm{IV}}$ less than $\cdot 5$.

It appears therefore that, in the case of $\alpha$ greater than $\cdot 5$, a small odd number of reciprocal transformations can hardly fail to detect the imaginary roots, supposing $\alpha_{n}$ always greater than $\cdot 5$.

Taking now the case a not greater than $\cdot 5$, we shall obtain the least value which $\beta_{1}$ can in this case hold, by making in it $\alpha=\cdot 5$; it becomes then

$$
\frac{\beta}{.25+\beta^{2}},
$$

which is precisely the same as the inferior limit to the value of $\beta_{11}$ in the preceding case: it follows therefore from what has been there shown, that if $\alpha$ be not greater than 5 the second reciprocal equation must detect the imaginary roots, unless $\beta$ be less than $\cdot 134$.

On a similar hypothesis the third reciprocal equation cannot fail unless $\beta_{1}$ be less than 134 , which involves the condition $\beta$ less than 033 : and so on.

In thus developing the changes which this limit of $\beta$ successively undergoes, it has been assumed that $\alpha_{n}$, the real part of the imaginary roots in the $n$th reciprocal equation, retains the character assigned to it in each particular case; but it is manifest that if it does not retain its character, the change will only have the effect of altering the hypothesis from $a>\cdot 5$ to $\alpha<\cdot 5$, or vice versá.

Independently of the additional value which these considerations give to the criterion of Budan, there is yet another most difficult case which the same operations tend to elucidate, viz. that in which two or more roots are nearly equal to one another. In fact, let $a$ and $b$ be two roots very nearly equal, both of them positive and less than unity, a condition always attainable; in the first reciprocal equation these roots will appear under the form $\frac{1}{a}$ and $\frac{1}{b}$, and their difference becomes $\frac{a-b}{a b}$, greater than before, since $a b$ is a proper fraction. Now to whatever extent the roots of this equation are diminished, their difference is unaltered; if therefore this difference should still be less than unity, another reciprocal transformation will again increase it; so that each transformation must of necessity 
bring us nearer to the point at which the roots corresponding to $a$ and $b$ are separable by means of unital reductions. When this point is arrived at, we are at once enabled to assign the true values of the roots by means of a continued fraction, similarly to the method employed by Lagrange, as the following example will show.

The given equation is

$$
x^{5}+7 x^{4}-144 x^{3}+611 x^{2}-928 x+362=0,
$$

from which we get successively,

$x_{1}^{5}+12 x_{1}^{4}-106 x_{1}^{3}+231 x_{1}^{2}-105 x_{1}-91=0 \quad\left(x_{1}=x-1\right)$

$x_{11}{ }^{5}+17 x_{11}^{4}-48 x_{11}^{3}-5 x_{11}^{2}+92 x_{11}-58=0\left(x_{11}=x-2\right)$

$x_{111}{ }^{5}+22 x_{111}{ }^{4}+30 x_{111}^{3}-37 x_{111}{ }^{2}+11 x_{111}-1=0\left(x_{111}=x-3\right)$

at the next transformation we shall evidently lose three variations; taking therefore the reciprocal equation and reducing, we have

$y_{1}{ }^{5}-6 y_{1}{ }^{4}+3 y_{1}{ }^{3}+25 y_{1}{ }^{2}-10 y_{1}-26=0\left(y_{1}=y-1, y=\frac{1}{x_{111}}\right)$.

Since this equation retains the three variations, there is every probability, by Budan's criterion, that the indicated roots are all real. Proceeding with the reductions, and retaining the same notation, we obtain

$$
\begin{aligned}
& y_{11}{ }^{5}-y_{11}{ }^{4}-11 y_{11}{ }^{3}+8 y_{11}{ }^{2}+30 y_{11}-13=0 \ldots 3 \text { variations. } \\
& y_{111}{ }^{5}+4 y_{111}{ }^{4}-5 y_{111}{ }^{3}-21 y_{111}{ }^{2}+14 y_{111}+14=0 . .2 \text { var. }
\end{aligned}
$$

so that there is a root of the equation in $y$ between 2 and 3 . In continuation, we have

$$
y_{\mathrm{rv}}{ }^{5}+9 y_{\mathrm{rv}}{ }^{4}+21 y_{\mathrm{rv}}{ }^{3}-2 y_{\mathrm{rv}}{ }^{2}-22 y_{\mathrm{rv}}+7=0 . .2 \text { variations, }
$$
and in the equation in $y_{\mathrm{v}}$ there will be no variations. Again, therefore, we take the reciprocal equation in $z=\frac{1}{y_{\mathrm{IV}}}$ and continue the reductions :

$$
7 z_{1}^{5}+13 z_{1}^{4}-20 z_{1}^{3}-47 z_{1}^{2}-8 z_{1}+14=0 \ldots 2 \text { variations; }
$$

from which it still appears that these roots are not imaginary. Proceeding as before, we get

$7 z_{11}^{5}+48 z_{11}^{4}+102 z_{11}^{3}+41 z_{11}^{2}-75 z_{11}-41=0 \ldots 1$ var. and the equation in $z_{113}$ will contain only permanences; one root therefore of the equation in $z$ is between 1 and 2 , and the other between 2 and 3.

In order to determine the actual approximate values of these roots in the original equation, we have, by making $y=2 \cdot 5$, $z=1 \cdot 5$, and $z=2 \cdot 5$, the three continued fractions, 
100 Mr.J.R. Christie on the Extensionoj Budan's Criterion, \&c.

$x_{111}=\frac{1}{2}+\frac{1}{2} \quad x_{111}=\frac{1}{4}+\frac{1}{1}+\frac{1}{2}^{x_{111}}=\frac{1}{4}+\frac{1}{2}+\frac{1}{2}:$

whence $x=3.4, x=3.214, x=3.227$.

As a still more difficult example of the separation of roots very nearly equal to one another, let us take the equation

$$
v^{5}-82 v^{4}+2404 v^{3}-26394 v^{2}+6132 v-360=0 \text {, }
$$

and it will be found that the following transformations will be obtained, viz.

$$
\begin{aligned}
& 360 w_{\mathrm{VIII}}^{5}+8268 w_{\mathrm{VIII}}{ }^{4}+60570 w_{\mathrm{VII}}^{3}+119564 w_{\mathrm{VIII}}{ }^{2} \\
& -156270 w_{\mathrm{VIII}}+40335=0 \ldots w=\frac{1}{v}, \\
& 40335 x_{11}^{5}+247080 x_{1 I}^{4}+482804 x_{11}^{3}+254274 x_{11}^{2} \\
& -88524 x_{\mathrm{II}}+6088=0 \ldots \ldots \ldots x=\frac{1}{w_{\mathrm{VII}}}, \\
& 6088 y_{\mathrm{VII}}^{5}+124556 y_{\mathrm{VII}}{ }^{4}+758712 y_{\mathrm{VII}}^{3}+678342 y_{\mathrm{VII}}{ }^{2} \\
& -3983874 y_{\mathrm{vII}}+24.18165=0 . . y=\frac{1}{x_{\mathrm{II}}}, \\
& 2418165 z_{1}^{5}+8106951 z_{1}^{4}+8924496 z_{1}^{3}+3072144 z_{1}^{2} \\
& -167665 z_{1}+1989=0 \ldots \ldots \ldots z=\frac{1}{y_{\mathrm{vII}}}, \\
& 1989 t^{5}-167665 t^{4}+3072144 t^{3}+8924496 t^{2}+8106951 t \\
& +2418165=0 \ldots \ldots \ldots \ldots \ldots t=\frac{1}{z_{1}} .
\end{aligned}
$$

Now it will be found that one root of this equation is between 30 and 31 , the other between 50 and 51, so that we have the continued fractions,

$$
v=\frac{1}{8+\frac{1}{2}+\frac{1}{7}+\frac{1}{1}+\frac{1}{30}+\frac{1}{2}} \quad v=\frac{1}{8}+\frac{1}{2}+\frac{1}{7}+\frac{1}{1+\frac{1}{50}+\frac{1}{2}}
$$

which give the values

$$
v=\cdot 118057 \text { and } v=\cdot 11805649 \text { : }
$$

the actual approximate roots to twelve places are

$$
\cdot 118056983866 \text { and } \cdot 118056440257 \text {. }
$$

The number of reciprocal transformations necessary to effect the separations of the roots, will of course primarily depend upon the number of figures in them which are identical; but there are certain points in the scale between 0 and 1 , from which, if the roots differ, in however small a degree, the one 
in excess and the other in defect, the first reciprocal equation suffices to show their inequality. These points are, evidently, the reciprocals of the integers above unity, that is

$$
\cdot 5, \quad \cdot 33, \quad \cdot 25, \quad \cdot 2, \quad \cdot 167, \quad \cdot 143,8 \mathrm{c} \text {. }
$$

It would at first sight appear that the least favourable case for the separation of the roots is that in which they differ least from unity, since this gives its maximum value to the denominator of the fraction $\frac{a-b}{a b}$; but it must be remarked that this hypothesis involves the consequence that the corresponding pair of roots in the reduced reciprocal equation will have their smallest value, and therefore be in the most favourable state for separation.

In order then to obtain some general insight into the extent of separation effected by each reciprocal transformation, we may take, as that of slowest divergence, the case in which the roots occupy a point midway between two of the above numbers, and are as near to 5 as this condition will allow. Assuming, therefore, the roots to be rather greater than 41 , we have $\frac{1}{a b}$ $=6$; and taking this number as the factor by means of which may be determined the divergence of two roots having, originally, their difference less than unity, it follows that the number of figures in its $n$th power will, not inaptly, represent the smallest number of figures which can be identical in the roots which become separable in the $n$th reciprocal equation.

From the preceding investigation we obtain a correct idea of the extreme rarity of the cases in which the impossibility, or the near equality of two or more doubtful roots can fail to be made manifest by means of this simple method of reciprocals; and the improbability of the occurrence of these cases affords the strongest evidence of the general utility of the method.

Royal Military Academy, June 13, 1842.

XVII. On the Analytical Condition of the Rectilinear Motion of Fluids. By the Rev. J. Challis, M.A., F.R.A.S., Plumian Professor of Astronomy and Experimental Philosophy in the University of Cambridge*.

TTHE mathematical reasoning which I gave in the April 1 Number of this Journal (S. 3. vol. xx. p. 281) respecting a new equation in hydrodynamics, led me by indirect considerations to the conclusion, that when $u d x+v d y+w d z$ is a

* Communicated by the Author. 